\title{
Early OA: point of no return or a chance for regenerative approaches
}

\author{
Peter Angele ${ }^{1,2} \cdot$ Henning Madry ${ }^{3} \cdot$ E. Kon ${ }^{4}$
}

Published online: 18 May 2016

(C) European Society of Sports Traumatology, Knee Surgery, Arthroscopy (ESSKA) 2016

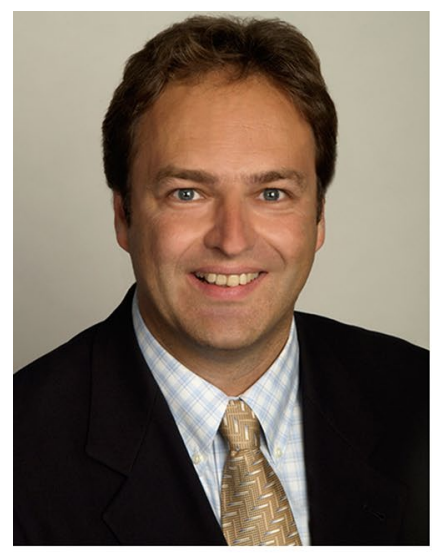

Peter Angele

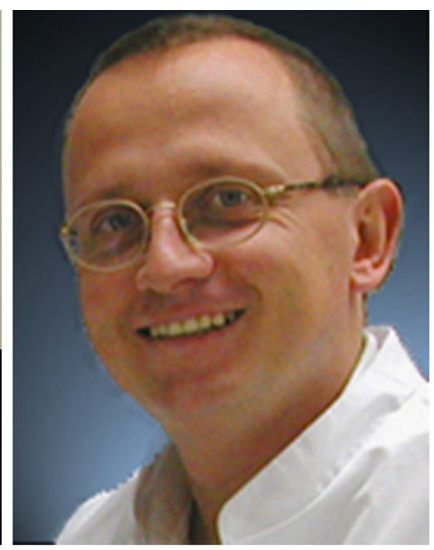

Henning Madry

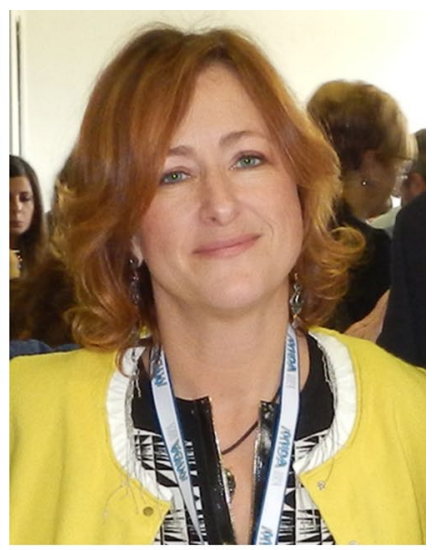

E. Kon
Osteoarthritis is one of the main burdens of the twenty-first century. It is associated with both the functional impairment of patients and enormous treatment costs for the healthcare system. For this reason, the diagnosis of "osteoarthritis"

Peter Angele

Peter.Angele@klinik.uni-regensburg.de

1 Department of Trauma Surgery, University Medical Centre Regensburg, Franz Josef Strauss Allee 11, 93042 Regensburg, Germany

2 Sporthopaedicum regensburg, Hildegard von Bingen Strasse 1, 93053 Regensburg, Germany

3 Lehrstuhl für Experimentelle Orthopädie und Arthroseforschung, Zentrum für Experimentelle Orthopädie, Universitätsklinikum des Saarlandes, Universität des Saarlandes, Gebäude 37, Kirrbergerstr. 100, 66421 Homburg, Germany

4 Biomechanics Lab - II Clinic, Rizzoli Orthopaedic Institute, Bologna, Italy offers immense potential for cost reductions for healthcare providers by narrowing the therapeutic spectrum. It appears that they favour - in our view, too early — end-stage therapy with the implantation of heavy metal in joints. Cost reductions can actually be achieved by healthcare providers by cutting reimbursements for conservative treatment and regenerative treatment approaches.

This leads to reduced treatment options for our patients. Regenerative treatment may perhaps not always regenerate healthy tissue, but it can perhaps delay the onset and clinical symptoms of advanced osteoarthritis. In particular, the change in lifestyle of our patients, with rising expectations of mobility, makes a clinical improvement for decades following meniscus allograft implantation, for example, worth the surgical treatment, even when advanced osteoarthritis cannot be prevented in the long term.

The regenerative treatment of chondral and osteochondral defects was designed to bring about the regeneration of localised traumatic lesions. Advanced osteoarthritis 
has always been regarded as a contraindication. However, where is the point of no return? How much degeneration can be accepted when implementing regenerative approaches? This special issue gives the present perspective on whether these approaches can also be used in "early OA" and, if they can, with which expected outcome.

The rapidly evolving new regenerative approaches make it necessary to demonstrate efficacy and effectiveness. It is up to us to conduct the appropriate studies. What is appropriate, however? For many years, we thought that only a randomised controlled trial was the optimum tool for proving the superiority of one treatment over another. However, we learned our lesson. A typical randomised controlled trial represents fewer than $4 \%$ of our patients in daily practice. We understand that we need all the different study levels in order to put the puzzle together to create a complete picture. Cohort and randomised clinical trials are necessary to test efficacy, but, to the same extent, register results are very useful for testing the effectiveness of a novel treatment. At present, our duty is to convince the healthcare authorities that randomised controlled trials are not always exclusively of value for outcome measurements.

In order to prove the efficacy of conservative and regenerative approaches, we also need to address differences in the underlying pathology of our melting pot of "osteoarthritis" or "early OA". The causes of OA are multifactorial. As a result, studies with the same or similar treatment approaches can result in opposite outcome results due to the diversity of the study cohorts. This diminishes our arguments in favour of supporting the unquestionable success of conservative or surgical regenerative approaches.

We therefore need to improve our grading and classification system and identify subgroups running a risk of varied outcome in order to obtain consistent results. This was one important stimulus for organising the ESSKA Consensus Meeting in Verona in 2015 entitled "Early OA: all around biological solutions". More than 350 clinicians and scientists met in order to clarify the term "early OA" from different points of view and discuss the treatment options.

One of the main steps was to find a classification for "early OA", which does not follow the rheumatology-based classification as a mild form of diffuse osteoarthritis. We felt it was important to create a classification, which could be useful for regenerative approaches.

The consensus also focused on the recognition of comorbidities which have to be treated in addition to cartilage treatment. Otherwise, a significant increase in failure rates for the regenerative approaches has to be expected.

The consensus gave a clarification of subchondral oedema in terms of nomenclature and treatment options. The impact of meniscus treatment options during "early OA" was reviewed. Load and overload were addressed, especially in an "early OA" situation.

The emphasis was also placed on the patellofemoral compartment. The outcome of cartilage regenerative approaches to focal traumatic defects of the patellofemoral compartment is significantly reduced to treating defects in the weight-bearing compartments. How far can we go during "early OA"?

In addition to established conservative and surgical regenerative treatment options, there is still room for improvement. The consensus highlighted new treatment approaches, including stem-cell therapies. The consensus also addressed the dependence of "early OA" and sports. Is there potential evidence that sport leads to "early OA"? In addition, how is the return to sport for patients with "early OA"? We hope that the consensus might lead to a better understanding of treatment options in "early OA" and responsible innovation and close collaboration among clinicians, scientific societies, industry and healthcare authorities in order to provide patients with the most suitable therapeutic solution. 